\title{
ACESSIBILIDADE DIGITAL NO PROGRAMA UM COMPUTADOR POR ALUNO: A EXPERIÊNCIA DA PARAÍBA
}

\author{
DIGITAL ACCESSIBILITY IN THE CONTEXT OF PROGRAM ONE \\ COMPUTER PER STUDENT: THE EXPERIENCE OF PARAÍBA
}

Mariano Castro Neto

Este artigo discute a acessibilidade digital no Programa Um Computador Por Aluno - ProUCA na Paraíba. Tratase de uma pesquisa aplicada e sua base empírica se deu a partir da seleção e identificação de aplicações digitais, potencializadoras de inclusão digital para alunos com deficiências, compatíveis com as limitações de hardware do laptop educacional. Inicialmente foi realizado levantamento do número de estudantes e tipos de deficiências e grau de dificuldades em relação à utilização do laptop educacional. Realizou-se o mapeamento, identificações e testagem das aplicações digitais livres, existentes no mercado, e ou em universidades. Como síntese final, e com base na análise de dados, pode-se afirmar que, das aplicações testadas, a que mais se aproxima dos critérios técnicos compatíveis com as limitações de hardware do laptop educacional é o Eviacam. Como continuação desta pesquisa, procura-se ampliar as discussões acerca da acessibilidade digital na concepção e implementação de políticas públiAcas voltadas à educação e inclusão digital. Em um Programa como o ProUCA.

Palavras clave: Acessibilidade Digital. Educação. Tecnologias e Sociedade. Projeto UCA..

This article discusses the digital accessibility in the One Computer Per Student Program in Paraíba's State, northeast of Brazil. The research applied and its empirical basis was based on the selection and identification of digital applications, potentials of digital inclusion for students with disabilities, compatible with the limitations of educational laptop hardware. |The survey was carried out on the number of students and types of disabilities and degree of difficulties in relation to the use of the educational laptop. The mapping, identifications and testing of free digital applications in the market and in universities were also carried out. Based on the data analysis, it can be said that, between the applications tested, the most approximates the technical criteria compatible with the limitations of educational laptop hardware is Eviacam. The continuation of this research aims to broaden the discussion about digital accessibility in the conception and implementation of public policies aimed at education and digital inclusion in a program like One Computer Per Student..

Keywords: Digital Accessibility. Education. Technologies and Society. UCA Project. 


\section{Introdução}

No período de 01/o3 a 31/12/2013 o Programa Um Computador por Aluno - ProUCA foi implantado em 11 (onze) escolas públicas municipais e estaduais da Paraíba. Participaram da formação continuada para a utilização pedagógica do laptop educacional 256 (duzentos e cinquenta e seis) professores e 11 gestores. Essas escolas foram selecionadas segundo critérios da Coordenação Pedagógica da UFPB em conjunto com a Secretaria Estadual de Educação, a União Nacional dos Dirigentes Municipais de Educação (Undime) e a Secretaria de Educação Básica do Ministério da Educação.

Tais ações foram desenvolvidas por meio de metodologias adequadas, que foram executadas com a cooperação da Universidade Federal da Paraíba, que tem larga experiência em projetos que envolvem as Tecnologias da Informação e Comunicação Digital (TICD) e, por esse motivo, contribuiu de forma significativa para ampliar os aspectos considerados na concepção do projeto, na elaboração da proposta de formação e na avaliação do mesmo. (RELATÓRIO FINAL DO UCA, 2014).

Do ponto de vista pedagógico, Programas como o ProUCA, na sua concepção, devem considerar aspectos de usabilidade, de acessibilidade digital que promovam inclusão para o maior número de alunos, com deficiências. Uma das dificuldades relatadas pelos professores, segundo Bezerra da Silva (2014) é tornar o laptop educacional acessível aos alunos com deficiências e promover a inclusão digital.

Nesse contexto, este artigo se propõe a apresentar soluções digitais para o laptop educacional, considerando suas limitações de hardware, para promover a acessibilidade digital dos alunos deficientes das 11 (onze) escolas contempladas com o ProUCA na Paraíba.

Com base nas revisões bibliográficas de MATOAN (2012), RENATO (2013), CAROLINY BATISTA (2013), CASTRO NETO, (2013) VENANCIO (2012), MAYOR (1994), dentre outros, se problematizou acerca da importância da acessibilidade digital nos laptops educacionais do ProUCA, e identificar soluções compatíveis com as características de hardware do laptop educacional.

\section{Processos ensino-aprendizagem na sociedade do conhecimento}

No contexto da sociedade do conhecimento mudanças no sistema educativo são imperativos. Vários países estão investindo na pesquisa em virtude da necessidade de adaptação. Esse investimento surge da necessidade de preparação da mão de obra que será exigida no futuro, o reflexo disso é a quantidade de programas de políticas públicas que primam por inserir recursos tecnológicos e midiáticos no contexto escolar.

Entretanto, as tecnologias de informação e comunicação digital sozinha não agregaram em nenhum aspecto à educação, se não houver um plano de ação, uma alteração metodológica que justifique o seu uso nas escolas de modo a provocar mudanças na prática docente.

Considerando que as tecnologias de informação e comunicação digital e seus recursos midiáticos apresentam atualmente possibilidades inimagináveis pela maioria das pessoas há algumas décadas. Hoje, porém a escola trabalha com um público capaz de fluir com desenvoltura entre as mídias e se adaptar as mudanças com mais facilidade de que se comparado com as gerações anteriores, são os nativos digitais, Presnky (2001: 1) faz algumas colocações importantes sobre este termo "[...] Como deveríamos chamar estes "novos" alunos de hoje? Alguns se referem a eles como N-gen [Net] ou D-gen [Digital]. Porém a denominação mais utilizada que eu encontrei para eles é Nativos Digitais. Nossos estudantes de hoje são todos "falantes nativos" da linguagem digital dos computadores, vídeo games e internet”.

O grande desafio para a educação centraliza-se na integração com a dimensão tecnológica, pois, a escola pretendida no século XXI necessita de mudanças significativas, de investimentos sistemáticos e de incentivos na execução do seu papel enquanto instituição fundamental para o desenvolvimento social do país. Libâneo (2004, p.51) explica que "A escola necessária para fazer frente a essas realidades é a que provê formação cultural e científica, que possibilita o contato dos alunos com a cultura, aquela cultura provida pela ciência, pela técnica, pela linguagem, pela estética, pela ética”. 
É com base nestas reflexões que este estudo problematizou acerca da importância da acessibilidade digital nos laptops educacionais do ProUCA, e identificar e implementar soluções compatíveis com o laptop educacional.

\section{Programa Um Computador por Aluno}

O Projeto Um Computador por Aluno tem como objetivo propiciar o desenvolvimento científico e tecnológico por meio da expansão do conhecimento, a fim de permitir a emancipação individual e coletiva, a consolidação da democracia, a melhoria da qualidade de vida e a equidade social amparada em valores éticos, estéticos e solidários (BRASIL, 2007).

Segundo o Ministério de Desenvolvimento, Indústria e Comércio - MDIC (2009) o projeto tem como pressupostos os seguintes itens: Mobilidade, pois poderá romper com o conceito de utilização pedagógica de equipamentos fixos de informática em um único ambiente, oportunizando a aquisição de novos conhecimentos a partir do uso de dispositivos em outros ambientes dentro e fora da escola; Conectividade, que juntamente com a mobilidade vai permitir a expansão do uso dentro e fora da escola, capaz de promover a conectividade de forma eficiente e abrangente tanto na escola como na comunidade de entorno; Baixo custo dos equipamentos, esta é condição primordial para a aquisição de uma grande quantidade de unidades e por fim, a Utilização dos Laptops deve servir para atividades pedagógicas e de gestão da escola;

Já os objetivos educacionais do projeto são: Contribuir na construção da sociedade sustentável mediante desenvolvimento de competências; Inovar os sistemas de ensino para melhorar a qualidade da educação com equidade no país; Ampliar o processo de inclusão digital das comunidades escolares; Possibilitar a cada estudante e educador da rede pública do ensino básico o uso de um laptop para ampliar seu acesso à informação e participar da construção coletiva do conhecimento; Conceber, desenvolver e valorizar a formação de educadores (gestores e professores) na utilização do laptop educacional com estudantes; Criar a rede nacional de desenvolvimento do projeto para implantação, implementação, acompanhamento e avaliação do processo de uso do laptop educacional.

O equipamento utilizado para o Projeto UCA possui largura e altura do equipamento nas dimensões de uma folha de papel A4 (21x29, $7 \mathrm{~cm})$, tela de cristal líquido (LCD) e dispositivos de segurança adequados. O equipamento permite acesso ao Programa de Formação Continuada em Mídias na Educação, tem baixo consumo de energia e autonomia suficiente para garantir o uso integral durante os turnos da escola. $\mathrm{O}$ equipamento permite ainda a reprodução de sons, vídeos, conexão com máquinas fotográficas digitais, filmadora digital, gravador de voz e vídeo (MDIC, 2009).

\section{O projeto UCA no estado da Paraíba}

Na Paraíba, a implantação do projeto acontece através da Universidade Federal da Paraíba, o mesmo foi iniciado em 2010, neste mesmo ano dez (10) escolas participaram da implantação no estado. Desde então estas escolas permaneceram integrando o projeto, a Tabela 1 apresenta as escolas distribuídas por cidade, bem como a quantidade de Laptops recebidos, e contingente de professores e gestores participantes do projeto, contudo, o modelo de formação aqui descrito iniciou-se em agosto de 2012.

\begin{tabular}{llll} 
Escolas UCA & Laptops & Municípios & Prof \\
EMEPA & 536 & Bananeiras & 36 \\
\hline EMEFTGP & 502 & Brejo do Cruz & 33 \\
\hline EEEFNSR & 800 & Campina Grande & 24 \\
\hline EMEFRFB & 500 & Campina Grande & 35 \\
\hline EEEFJS & 359 & Catolé do Rocha & 26 \\
\hline EEEFDBM & 255 & João Pessoa & 24 \\
\hline EMEIEFAMS & 285 & Nazarezinho & 18 \\
\hline EMEFZM & 166 & Patos & 12 \\
\hline EEEFFB & 303 & Sapé & 20 \\
\hline EEEFMMP & 300 & Vieirópolis & 14 \\
\hline TOTAL & 4.006 & o9 & 242 \\
\hline
\end{tabular}

Tabela 1 - Escolas Integrantes do UCA Paraíba. Fonte: Bezerra da Silva, 2014 
Ocorreu no período de agosto a dezembro de 2012 três encontros presenciais em João Pessoa como parte da formação da fase I. Nessa fase, realizou-se a migração do sistema operacional Metasys para o Ubuntu em todos os laptops educacionais das escolas Paraibanas. Criou-se o blog (link x - blind review) como espaço de socialização de experiências didáticas bem sucedidas entre as escolas participantes do projeto.

Essa experiência possibilitou à equipe de formadores em reunião de trabalho discutir acerca da importância da continuidade do projeto UCA/PB sob vários aspectos. Um deles trata-se de ampliar a oferta do curso de formação do uso pedagógico do laptop educacional para todos os professores, gestores e alunos monitores das dez escolas participantes do projeto, além dos profissionais dos NTE/M.

Nesse período, a equipe de formadores solicitou a continuidade da Fase II do projeto-piloto UCA. $\mathrm{Na}$ fase II houve uma reformulação do modelo de formação, na busca pela inclusão de metodologias e conteúdos adequados, desenvolvidos por uma equipe de seis formadores, todos, professores da Universidade Federal da Paraíba. A abordagem metodológica desta formação fundamenta-se nos seguintes princípios teóricos: i) Tecnológico (apropriação dos recursos tecnológicos voltados para o uso do sistema Linux Educacional e de aplicativos existentes nos laptops educacionais); ii) Pedagógico (uso dos laptops nos processos de ensinar e aprender, na gestão de tempos, espaços e relações entre os protagonistas da escola, do sistema de ensino e da comunidade externa); iii) Teórico (articulação de teorias educacionais que permitam compreender criticamente os usos em diferentes contextos e reconstruir novas práticas pedagógicas e de gestão).

Nessa perspectiva, além das ações constantes no projeto original, propõe-se o desenvolvimento de três ações:

A ação 1 se refere à preparação dos formadores, tutores, apresentação do modelo de formação, o mesmo divide-se nas seguintes fases: Fase 1: Apro- priação tecnológica - 4oh (presencial); Fase 2: Introdução ao Projeto UCA e planejamento da formação na escola - 24h (16h presenciais e $8 \mathrm{~h}$ à distância, em 1 semana); Fase 3: Formação na escola - 18oh (a distância com reuniões presenciais); Fase 4 - Avaliação, depuração e reestruturação da formação - 16h (presenciais).

A ação 2 trata da formação dos professores, gestores das escolas e equipe do NTE/M a ser realizada pela equipe de Formação do ProUCA / UFPB. A execução dessa ação está estruturada em encontros presenciais e virtuais por meio da plataforma Moodle, perfazendo 150 horas (120h para módulos virtuais e 30 horas presencias - na escola e encontros globais em João Pessoa);

Os módulos trabalhados na ação 2 da formação continuada são os seguintes: Módulo I - Apropriação tecnológica e compreensão da proposta UCA - 4oh (presencial), módulo II - Web 2.0 - 3oh (distância), módulo III - Formação na escola proposta da ação - 4oh (distância), módulo IV - Análise e depuração da proposta de ação e elaboração do Projeto UCA da Escola (na gestão e prática pedagógica) - 40h, e módulo V - Seminário para apresentação dos resultados, análises com base em documentos e nas apresentações do projeto UCA da Escola - 72h.

\section{Método}

Inicialmente foi realizado levantamento do número de alunos regularmente matriculados e, desses, quantos deficientes, deficiências, necessidades e dificuldades em relação à utilização do laptop educacional, no que se refere à acessibilidade digital. Após essa etapa, realizou-se pesquisa bibliográfica em bancos de dados específicos da área para identificar trabalhos correlatos. A partir dessas fases identificaram-se aplicações livres existentes no mercado e ou em universidades e ou centros de pesquisas compatíveis com as limitações de hardware.

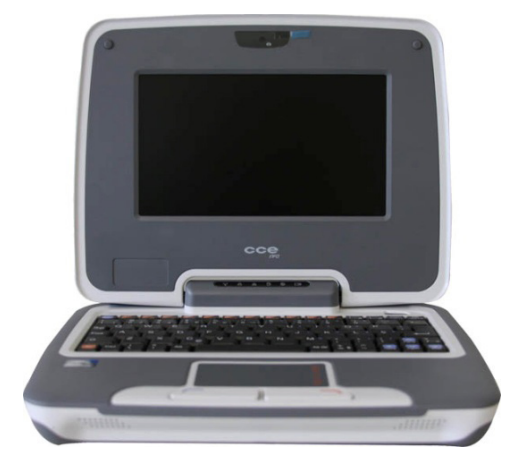

Figura 1 - Classmate PC. Fonte: http://www.cceinfo.com.br/uca/. 
pesa aproximadamente $1,3 \mathrm{~kg}$, processador Intel Atom N270, 1.6 giga-hertz de ciclos de clock, 4 GB e 512 megabytes de memória interna, tela de 7 polegadas em cristal líquido, webcam de 0.3 megapixels.

O Sistema Operacional o UbuntUCA, customizado especialmente para o laptop pelo professor motivador Gedimar Pereira, coordenador do UCA na Escola de Ensino Fundamental Padre Theodoro Becker,
Brusque, Santa Catarina. Com base na configuração do laptop educacional, realizaram-se pesquisas para identificar aplicações digitais compatíveis com essas limitações técnicas. Foram realizadas várias pesquisas bibliográficas no intuito de identificar aplicações digitais compatíveis com o UCA. A tabela 1 mostra a lista de softwares selecionados (Gok-Gnome, Orca, Enable Viacam ou simplesmente Eviacam, Mouselupa e Compiz) para compor os testes no Classmat PC.
Software $\quad$ Breve descrição das ferramentas

Gok-Gnome

Ferramenta de acessibilidade desenvolvida por: Miguel de Icasa, cujo objetivo é tornar acessível as funcionalidades do teclado e o mouse convencionais.
Orca

Software que promove acessibilidade, desenvolvido pelo Escritório do Programa de Acessibilidade da Sun Microsystems, Inc., cujo objetivo é a litura da tela do sistema, utilizado por deficientes visuais.

Enable Viacam

Ferramenta de acessibilidade, desenvolvido pelo CREA Software Systems, cujo objetivo é substituir o mouse convencional, controlando a localização do mesmo.

Software que promove acessibilidade, desenvolvido pelo grupo de pesquisa IMAGO ${ }^{\mathbf{1}}$, focado para o grupo de deficientes possuidores de baixa visão.

Ferramenta que promove acessibilidade, desenvolvida por: David Reveman, Sam Splis-

Compiz bury, Danny Baumann, Dennis Kasprzyk, Daniel van Vugt, indicada para deficientes visuais.
1 Grupo de

pesquisa do

Departamento de

Informática (DInf)

da Universidade

Federal do Paraná.

\section{Trabalhos Relacionados}

Em relação a estudos secundários (Mapeamentos e Revisões Sistemáticas) que abordem a acessibilidade digital Educativa no Ensino Fundamental e Médio em Escolas Estaduais. Realizou-se pesquisa nos Anais do Simpósio Brasileiro de Informática na Educação (SBIE) e o Workshop de Informática da Educação (WIE) nos últimos 3 anos.

Para melhor fundamentar a proposta aqui apresentada, realizou-se uma busca por trabalhos presentes na literatura que relatassem as experiências dos envolvidos com o Projeto UCA, em seu estado ou em seus municípios, de acordo com as funções exercidas dentro do projeto. Dentre o trabalhos relacionados destacaram-se alguns que serão brevemente apresentados a seguir.

Silvia e Susin (2012) apresentam em seu trabalho, a importância do uso das tecnologias de informação e comunicação dentro do âmbito escolar, mas especificadamente no processo de ensino aprendizagem na disciplina de Ciências do Ensino Fundamental. Neste trabalho, os autores discutem os efeitos da inserção dos laptops em uma instituição incluída no projeto, analisando o seu uso por alunos. A análise foi feita através de observações dos participantes realizadas em aulas de Ciências e em projetos de investigação, como resultados obteve- 
se a ênfase o da importância da interatividade e da colaboração nas atividades propostas, e da possibilidade disto mediante a utilização de computadores portáteis.

Pretto et al (2012) apresenta uma pesquisa em andamento que entre outras questões, discute situações inerentes ao processo de implementação das ações do projeto em dez escolas públicas do estado da Bahia, assim como apresenta reflexões sobre as necessidades de reestruturação, reorganização e diálogos interinstitucionais necessários à efetivação das ações previamente estabelecidas para o ProUCA. Os autores apresentam o modelo de implantação de tecnologia nas escolas chamado de "modelo 1:1" além de identificarem que os problemas que surgem durante a implantação do projeto são reincidentes.

Gonçalves (2012), partindo da perspectiva do uso do computador cada vez mais frequente nas salas de aula por professores e alunos, como um recurso muito importante para educação, apresenta a necessidade de se refletir sobre a utilização do computador como uma ferramenta pedagógica para subsidiar a aquisição de conhecimento no ambiente escolar. Para que isso fosse possível, foi feita uma pesquisa com critérios qualitativos, baseada em observações feitas nas turmas do $1^{\circ} \mathrm{e} 2^{\circ}$ ano do Ensino Fundamental da escola Classe 102 do Recanto das Emas - Distrito Federal. A pesquisa foi realizada com o auxilio de estudantes do curso de Pedagogia da Faculdade de Educação - FE da Universidade de Brasília - UNB. Como resultado observou-se um desenvolvimento cognitivo satisfatório do alunado.

Spagnolo et al. (2012), apresenta em seu trabalho um análise feita através de relatos de professores, a experiência no âmbito de aplicação do Projeto Um Computador por Aluno - ProUCA na Escola Municipal de Ensino Fundamental Caminhos do Aprender do município de Fagundes Varela/RS em parceria com Faculdade de Educação da Universidade de Passo Fundo. O estudo concluiu que os cursistas (professores) se sentiram muito entusiasmo com a capacitação e o intuito de promover formas de apropriação dos recursos tecnológicos e das inúmeras possibilidades de interação e inovação nas práticas escolares, mas, ao mesmo tempo demonstram-se preocupados com enorme desafio de assegurar uma educação de qualidade diante dos novos paradigmas que surgem nesse processo.

Os trabalhos citados refletem, de modo geral, o estado da arte atual em torno das pesquisas sobre o projeto UCA. A maioria dos trabalhos relacionados ao projeto apresenta as suas ações ou discutem brevemente os seus resultados em determinado local, raros são os trabalhos que discutem a influência do projeto para a equipe formadora, neste sentido, o presente artigo se dedica a abordar as experiências vivenciadas pelos tutores virtuais, e o modo como eles contribuíram para o desenvolvimento do projeto e como o projeto possibilitou o desenvolvimento de suas capacidades pedagógicas, visto que, os tutores virtuais do ProjetoUCA-PB são graduandos em Licenciatura em Ciência da Computação, curso oferecido pela Universidade Federal da Paraíba, com o intuito de formar profissionais para atuarem no processo de ensino aprendizagem e, principalmente, nas mudanças de paradigmas diante das possibilidades oferecidas pelas tecnologias de comunicação digital emergentes no contexto escolar.

Realizaram-se pesquisas acerca de trabalhos relacionados a Acessibilidade no Programa Um Computador por Aluno no Ensino Fundamental em Escolas Públicas nos Anais do CBIE, nas edições de 2013/2014/2015. Os artigos selecionados tratam de estudos pontuais. No caso de Acessibilidade Digital no ProUCA as pesquisas ainda são incipientes. Como forma de contribuir para o debate, este artigo se dedica a problematizar acerca da importância de políticas públicas que assegure o cumprimento de critérios de acessibilidade digital, ergonômicos e pedagógicos em programas como o ProUCA.

\section{Discussão}

O Gok-Gnome apresenta como principais funcionalidades a manipulação, a estabilidade e a internacionalização. Possui suporte à acessibilidade de primeira classe. É baseado na política de Software Livre e Código Aberto, podendo os desenvolvedores contribuírem para melhorar sua qualidade e funcionalidades, possui as opções que usuários de computadores necessitam para um ambiente moderno, suas funcionalidades como teclado e mouse virtuais, são adaptadas para deficientes que por algum motivo não podem utilizar o teclado e/ou mouse convencionais.

Como mostra a Figura 2, o teclado virtual do GokGnome dispõe das funcionalidades de ocultar o teclado, ou seja, o mesmo pode ocultar-se para que o usuário possa observar o texto disposto por trás da interface do software, possui opções de mover o curso para movimentar-se em todas as direções: cima, baixo, lado esquerdo e lado direito, as teclas de talho como Ctrl + C (copiar) é substituída por uma simples tecla, dentre outros atalhos. Enfim, substituem de maneira precisa todas as funcionalidades do teclado convencional. 


\begin{tabular}{|c|c|c|c|c|c|c|c|c|}
\hline Voltar & a & b & c & d & e & $f$ & & \\
\hline $\mathbf{h}$ & i & j & k & I & $\mathbf{m}$ & $\mathbf{n}$ & & \\
\hline $\mathbf{p}$ & q & $r$ & $\mathbf{s}$ & $\mathrm{t}$ & $\mathbf{u}$ & $\mathbf{v}$ & & \\
\hline$x$ & y & $\mathbf{z}$ & 1 & 2 & 3 & 4 & & \\
\hline 6 & 7 & 8 & 9 & $\mathbf{0}$ & - & $=$ & & \\
\hline$]$ & ; & ' & $\#$ & 1 & , & . & & \\
\hline shift & Nível 2 & Nível 3 & $\begin{array}{l}\text { Back } \\
\text { snace }\end{array}$ & Tab & \multicolumn{2}{|c|}{ Barra de espaço } & Enter & • \\
\hline \multicolumn{3}{|c|}{ Edluar } & \multicolumn{3}{|c|}{$\begin{array}{l}\text { Num } \\
\text { nad }\end{array}$} & \multicolumn{3}{|c|}{ Ocultar } \\
\hline
\end{tabular}

Figura 2 -Teclado virtual da ferramenta Gok-Gnome. Fonte: print screen da aplicação no sistema operacional Ubuntuca.

As funcionalidades do mouse virtual, como mostra a Figura 3, encontradas na ferramenta acessível Gok-Gnome, são de deslocamento do cursor em todas as direções: diagonal superior à esquerda, diagonal inferior à direita, cima, baixo, esquerda, direita, dentre outras direções, ainda dispões de funcionalidades como travar o cursor, clicar nos botões de confirmação, de acesso ao menu.

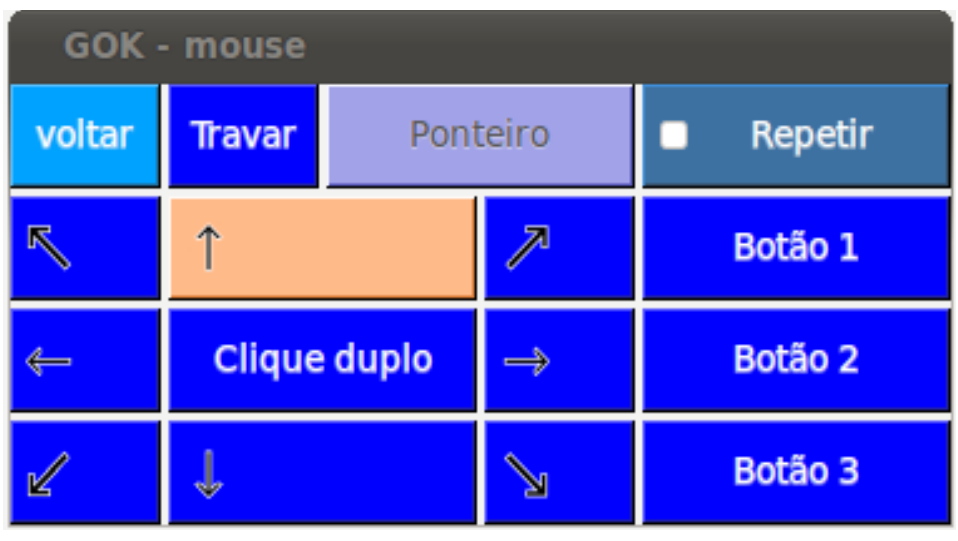

Figura 3 - Mouse virtual da ferramenta Gok-Gnome. Fonte: print screen da aplicação no sistema operacional Ubuntuca.

A Figura 3 mostra um leitor de tela para pessoas com deficiência visual, também uma ferramenta com código aberto, permitindo que qualquer programador faça alterações em suas funcionalidades, utiliza várias combinações de síntese, como o Braille, a fala e a ampliação de conteúdo. Sua interface gráfica de usuário, do inglês Graphical User Interface - GUI - permite ao usuário personalizar todas as necessidades apontadas antes.
O Enable Viacam, conforme mostra a Figura 4 é um software baseado na política de Software Livre, tem o objetivo de captar os movimentos realizados pela cabeça do usuário, por meio da webcam, substitui os movimentos realizados pelo mouse. Possui vantagem por ser compacto considerado muito eficiente, pois não perde a calibragem. É indicado para usuários que possuem necessidades especiais como: visuais, auditivas, físicas, de fala, cognitivas e neurológicas. 


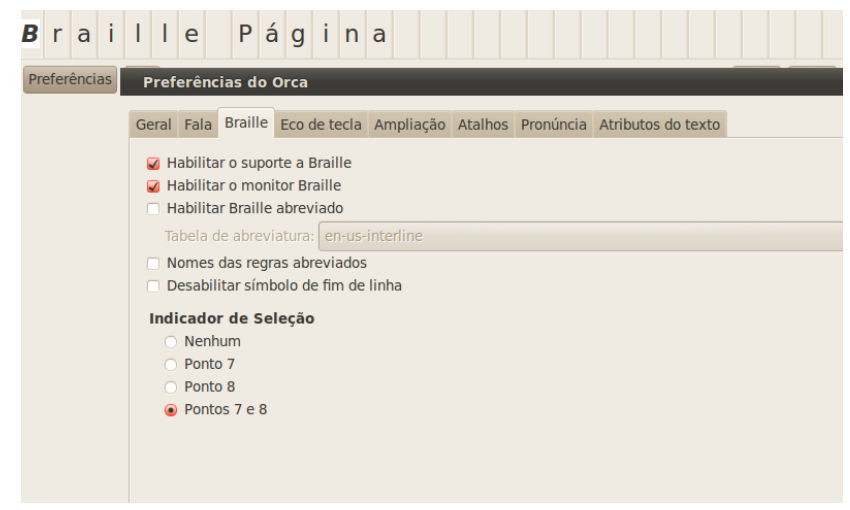

Figura 4 - Tela de configuração do Orca. Fonte: print screen da aplicação no sistema operacional Ubuntuca..

De acordo com a Figura 4, o usuário pode configurá-lo de acordo com sua necessidade, como fala (o sistema soa tudo que está descrito na tela do computador), braile (preferências de ativação do teclado), eco de tela (é possível habilitar de acordo com a necessidade do usuário), ampliação, atalhos, pronuncia, entre outros, uma ferramenta perfeita para deficientes visuais.

Mouselupa trata-se de um software que amplia os conteúdos digitais contido na tela do sistema, assim como a navegação pela internet, permite e facilita a utilização do computador por pessoas que possuem baixa visão. Segue as normas da GNU
(General Public License) de acessibilidade; essa ferramenta foi aperfeiçoada nos Projetos: Vision Linux e Linux Acessível, financiado pela Fundação Araucária, CNPq e FINAP.

Uma das funcionalidades do Mouselupa, conforme mostra a Figura 5, é a ampliação do conteúdo na tela em 4.ox e 40opx (podendo variar) de diâmetro em formato circular, já no formato em quadrado a ampliação é de 12.0x e 60opx (podendo variar) de lado, pode-se observar que ao lado esquerdo da imagem, encontra-se a interface, por sua vez bem intuitiva para que o usuário possa ampliar e diminuir o conteúdo de acordo com sua necessidade.

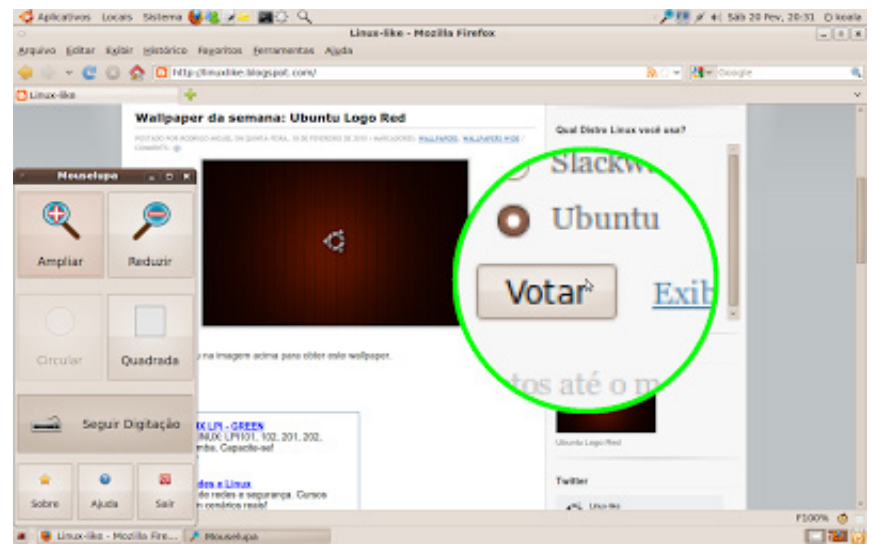

Figura 4 - Interface do software Mouselupa. Fonte: http://linuxlike.blogspot.com.br/2010/o2/mouselupaacessibilidade-para-pessoas.html

O software Compiz, cuja funcionalidade deixa o ambiente mais interessante, pois o mesmo é um gerenciador de telas que permite surtir efeitos e animações, resultando em um visual mais rico e, em muitos casos, mais proveitoso. A quantidade de efeitos visuais é enorme, por isto é necessário um gerenciador para poder alterná-las corretamente.

Todo o processo de testificação foi realizado por partes, primeiro foi instalado o teclado virtual Gok-Gnome, que teve o resultado esperado no 
procedimento de instalação e de execução, porém, ao utilizar a ferramenta, foi possível perceber que o teclado virtual da mesma, ocupa uma parte significativa da tela, impedindo que o digitador visualize o texto por trás da interface do software, mas há a opção de "ocultar" o teclado, que minimiza essa dificuldade, podendo o usuário ampliar ou diminuir a interface.

Da mesma forma com o Orca, foi sua instalação e execução com êxito, porém, ao utilizá-lo, foi possível notar que a interface ocupa toda parte da tela e não foi possível minimizá-la, só se pode sair da supracitada ferramenta para acessar outros programas, através de atalhos usando as teclas Alt + Tab (alternar telas), pois, através da barra menu "Leitor e ampliador de tela do Orca", o cursor do mouse não consegue acessar a opção de minimizar a tela, pois a todo o momento a mesma aparece, porém quando se aponta o cursor para acessá-la, a barra de menu desaparece.
Com os testes realizados na ferramenta Eviacam, foi possível instalá-la e executá-la com eficácia, nem o sistema operacional, nem tão pouco o hardware, demostraram quaisquer limitações ou erros. Nesse sentido, por meio do menu do software Eviacam é possível configurar os movimentos, limite da área de espaços, eventos de clique, ativação das teclas de atalho do teclado, entre outros, ainda possui a funcionalidade de travar o cursor, esta funcionalidade é indicada para deficientes que possuem movimentos involuntários.

Ao instalar as outras ferramentas acessíveis, foram contatados vários erros. O Compiz apresentou um bug na etapa de instalação, o qual impossibilitou a realização do processo citado e, sequencialmente, a execução do aplicativo, como o $\log ^{2}$ de erro descrito abaixo no Quadro 1, foi "encontrado" algumas extensões de arquivos já instalados, que impossibilita tal ação, trata-se de um bug do Metasys (antigo sistema operacional) que ainda é repercutido na versão 3 do UbuntUCA.
2 É exibido na tela da IDE para identificação da causa do erro.

\author{
aluno@: \$su \\ Senha: \\ compiz (core) - Warn: SmcOpenConnection failed: None of the authentication protocols specified are sup- \\ ported \\ compiz (core) - Fatal: Software rendering detected. \\ compiz (core) - Error: Failed to manage screen: o \\ compiz (core) - Fatal: No manageable screens found on display :0.0 \\ Launching fallback window manager \\ (metacity: 1200): Gtk-WARNING **: Não foi possível localizar a ferramenta de temas no module_path: \\ "pixmap"... \\ metacity:ERROR:core/prefs.c:2495:meta_prefs_get_workspace_name: assertion failed: (workspace_ \\ names[i] != NULL) \\ Abortadoroot@:/home/aluno\#
}

Quadro 1 - Log de erro do software Compiz. Fonte: Shell do Sistema Operacional Ubuntuca.

Outro software que não foi possível instalar para compor os testes no Classmate PC e consequentemente no Sistema Operacional foi o Mouselupa. O Shell do UbuntUCA informa que não foi possível instalar o Mouselupa, pois há uma versão do software instalada anteriormente, mas quando se faz uma pesquisa (execução) sobre o mesmo não é encontrado registros. Após várias verificações, foi constatado que não há extensões do mesmo, tudo leva a crer que seja um bug do sistema.
Foi constatado limitação de hardware após instalar o software Gok-Gnome e o Orca, o Sistema Operacional executou as tarefas corretamente, mas, em seguida, ao tentar instalar a ferramenta Eviacam, foi apresentada mensagem que retrata limitações de capacidade de armazenamento em disco, o qual o Sistema Operacional mostrou-se incapaz de instalar tal ferramenta. A solução encontrada para resolver este problema foi desinstalar o GokGnome e subsequente instalar o Eviacam. 
A tabela 3 mostra os resultados dos testes de instalação e execução das aplicações digitais ou ferra- mentas de acessibilidade selecionadas para compor os testes.

\begin{tabular}{clllll}
\multirow{2}{*}{ SO } & Gok-Gnome & eViacam & Orca & Compiz & Mouselupa \\
\cline { 3 - 5 } & Possível & Possível & Possível & Possível & Não possível \\
\cline { 2 - 6 } & Executável & Executável & Executável & Não executável & Não executável \\
& & & & & \\
\end{tabular}

Tabela 3- Testes de Instalação das ferramentas de acessibilidade.

Dentre as aplicações digitais pesquisadas, 3 (três) são compatíveis com as características de hardware do laptop educacional, conforme mostra a tabela 2.7.

\section{Conclusão}

O desenvolvimento desta pesquisa permitiu identificar algumas aplicações digitais compatíveis com as limitações do laptop educacional e que podem promover a acessibilidade digital. Permitiu ainda discutir a importância da acessibilidade digital no âmbito do Programa um Computador por Aluno ProUCA, no Estado da Paraíba, além de identificar, no contexto escolar, algumas aplicações digitais compatíveis com as configurações de hardware do laptop educacional.

Este estudo trouxe para o centro do debate a importância da acessibilidade digital, como requisito fundamental para a concepção de políticas públicas voltadas à educação com ênfase na inclusão digital de alunos de escolas públicas. De outro lado, os resultados mostram que temos imperativo repensar o conceito de infraestrutura tecnológica nas escolas, formação continuada dos professores e gestores quando se trata de inclusão digital.

É possível afirmar, em certa medida, que é imperativo criar mecanismos para assegurar a participação de profissionais de diversas áreas nas fases de concepção, desenvolvimento, implementação e avaliação de um programa dessa dimensão e que tenha como foco o uso de te tecnologias de informação e comunicação digital para escolas do ensino fundamental e públicas. Dentre as contribuições deste estudo, destacam-se:

a. discutir a importância da acessibilidade digital na concepção de políticas públicas voltadas a educação e inclusão digital;

b. identificar problemas técnicos "crônicos" das ferramentas: Gok-Gnome, eViacam, Orca, Compiz e Mouse lupa;

c. identificar algumas aplicações digitais compatíveis com as limitações de hardware do laptop educacional potencializadoras de processos de ensino-aprendizagem.

Como síntese final, e com base na análise de dados, pode-se afirmar que, das aplicações testadas, a que mais se aproxima dos critérios técnicos compatíveis com as limitações de hardware do laptop educacional é o Eviacam. Como continuação desta pesquisa, procura-se ampliar as discussões acerca da acessibilidade digital na concepção e implemen tação de políticas públicas voltadas à educação e inclusão digital. Em um Programa como o ProUCA. 


\section{Referências}

BEZERRA DA SILVA, Maciel. Acessibilidade digital e o programa um computador por aluno - ProUCA na Paraíba. 2014. 66F. Trabalho de Conclusão de Curso (Curso de Licenciatura em Ciência da Computação). Universidade Federal da Paraíba, UFPB, Campus IV - Rio Tinto Paraíba - Brasil, julho de 2014.

BRUNO, Horta Marques. Et al, Análise do Projeto de Lei n⿳0 2126/2011 na perspectiva da liberdade do uso da internet no Brasil. 2013.

CASTRO NETO, Mariano. Da teoria da atividade docente em ambientes virtuais de apoio à aprendizagem. 2006. 214f. Dissertação (Doutorado em Engenharia de Produção). Universidade Federal de Santa Catarina, UFSC, Florianópolis, Ilha de Santa Catarina - Brasil, Outubro/2006.

HIPERMÍDIA e acessibilidade na era da inclusão / Luciane Maria Fadel, Vania Ribas Ulbricht, Mariano Castro Neto. - João Pessoa: Ideia, 2013.

\section{MANTOAN, Maria Teresa Eglér. Visão informativa semestral do lar das moças cegas} (LMC), Ano 6-No 10-Agosto de 2012.

MAYOR, Federico. Declaração de Salamanca e enquadramento da ação, na área das necessidades educativas especiais. Conferência Mundial Sobre Necessidades Educativas Especiais Acesso e Qualidade. Salamanca, Espanha, 7-10 de junho de 1994.

Diretrizes Nacionais para a Educação Especial na Educação Básica. Ministério da Educação Secretária de Educação Especial - MEC; SEESP, 2001. 79 p.

RENATO, Marquezan Pereira. A trajetória diante do projeto Um Computador por Aluno (UCA): uma análise da Escola Municipal Vitor Miguel de Souza - Fpolis, 2013. (TCC). UFSC - Curso de Especialização em Educação Integral.

VENANCIO, V.; CORREA, A.G.D.; TELLES, E.O.; CORREA, C.M.; GARCIA, B.V.R.; RIBEIRO, C.C.; FICHEMAN, I.K.; LOPES, R.D. Integração dos Laptops ducacionais ao Cotidiano Escolar no UCA São Paulo: facilidades e dificuldades. In: I Congresso Brasileiro de Recursos Digitais na Educação, São Paulo, 2012.

ALVES, Fábio Pereira; MACIEL, Cristiano. A gamificação na educação: um panorama do fenômeno em ambientes virtuais de aprendizagem. 2014.

BARBOSA, F. C., ALVES, D., MENEZES, D., ALEXANDRE, M., CAMPOS, G., NAKAMURA, Y, LOPES, C. Robótica Educacional em Prol do Ensino de Matemática. In: Anais do Workshop de Informática na Escola. Vol. 21, No. 1, p. 271, 2015.

BECK, John C.; WADE, Mitchell. Got game: How the gamer generation is reshaping business forever. 2004. 
BENITTI, F. B. V., VAHLDICK, A., URBAN, D. L., KRUEGER, M. L., HALMA, A. Experimentação com Robótica Educativa no Ensino Médio: ambiente, atividades e resultados. In: Anais do XXIX Congresso da Sociedade Brasileira de Computação, Bento Gonçalves/RS p. 1811-1820, 2009.

CAMBRUZZI, E, DE SOUZA, R. M. Robótica Educativa na aprendizagem de Lógica de Programação: aplicação e análise. In: Anais do Workshop de Informática na Escola. Vol. 21, No. 1, p. 21, 2015 .

CHELLA, M. T. Ambiente de Robótica Educacional com Logo. In: XXII Congresso da Sociedade Brasileira de Computação - SBC2002. Florianópolis, 2002.

CRESWELL, J. W. Procedimentos qualitativos. Projeto de pesquisa: métodos qualitativo, quantitativo e misto, 2, 32-33. 2007.

DETERDING, S., DIXON, D., KHALED, R., NACKE. L. From game design elements to gamefulness: defining gamification. In Proceedings of the 15th International Academic MindTrek Conference: Envisioning Future Media Environments. NewYork: ACM. p. 9-15, 2011.

FARDO, Marcelo Luís. A gamificação como estratégia pedagógica: estudo de elementos dos games aplicados em processos de ensino e aprendizagem. 2014. Tese de Doutorado.

IBGE. Senso Demográfico. 2015. Disponível em:<http://www.ibge.gov.br>. Acesso em: 10 mar. 2016.

MCGONIGAL, Jane. Reality Is Broken: Why Games Make Us Better and How They Can Change The World. Nova Iorque: The Penguin Press, 2011. 